\title{
Un cas de kyste épidermoïde du plancher buccal
}

\section{Case report of an epidermoid cyst at the floor of the mouth}

AHMED ELFAHSI, ABDELILAH OUJILAL, MUSTAPHA LAHLOU, MOHAMED BOULAICH, MOHAMED KZADRI

\section{RÉSUMÉ}

Le kyste épidermoïde du plancher buccal est une tumeur bénigne et rare. Histologiquement, la paroi kystique est constituée par un épithélium malpighien pluristratifié, kératinisant, et le contenu de la cavité est composé par des squames de kératine. L'aspect histologique est bien différent de celui des autres kystes de type dermoïde ou tératoïde.

C'est un patient âgé de 23 ans qui présentait une tuméfaction du plancher buccal et de la région sus-hyoïdienne. D'évolution lente, cette tuméfaction était à l'origine de troubles de l'élocution et de la mastication. L'échographie, complétée par l'examen tomodensitométrique, a mis en évidence une masse kystique, échogène et bien limitée. Le traitement a consisté en une exérèse chirurgicale par voie endobuccale ; l'aspect macroscopique et l'examen histologique ont permis de préciser qu'il s'agissait d'un kyste épidermoide.

Les différents caractères de ce cas sont identiques à ceux retrouvés dans la littérature. (Med Buccale Chir Buccale 2006; 12: 175-8).

mots clés: kyste dermoïde, kyste épidermoïde, plancher buccal

médecine buccale chirurgie buccale

VOL. $12, \mathrm{~N}^{\circ} 3$ 2006

page 175

\section{SUMMARY}

Dermoids cysts of the oral floor are rare benign tumours, who having three histological aspects: dermoid, teratoid, and epidermoid. This one is characterized by the presence of a squamous stratified epithelium with cutaneous remnants.

We report a case of an epidermoid cyst of the oral floor, on a 23 years old man, who presented a submental mass increasing volume, generating progressively difficulty of elocution and chewing. The ultrasonography completed by a CT-scan, showed a cystic mass, echogene and well limited. The treatment consisted in a surgical excision. The features of this observation are identical to those of the other cases returned in the literature. (Med Buccale Chir Buccale 2006; 12: 178-8).

key words: dermoid cyst, epidermoid cyst, oral floor 
Le kyste épidermoïde est une tumeur bénigne d'origine embryonnaire. Sa localisation dans le plancher buccal est rare. II se présente alors cliniquement comme une tuméfaction sous la langue qui est refoulée, ou parfois sous-mentale donnant un aspect en double menton. Le diagnostic peut être évoqué sur l'examen clinique et le bilan radiologique mais il est confirmé par l'anatomopathologie. Nous rapportons un nouveau cas de kyste épidermoïde du plancher buccal afin de mettre l'accent sur ses caractéristiques épidémiologiques, cliniques et thérapeutiques.

\section{OBSERVATION CLINIQUE}

M. H. J, âgé de 23 ans, est hospitalisé en décembre 2003 dans le service pour une tuméfaction du plancher buccal, évoluant depuis trois ans mais qui a aug-

médecine

buccale

chirurgie

buccale

VOL. $12, \mathrm{~N}^{\circ} 3$ 2006

page 176 menté progressivement de taille. L'examen endobuccal révèle, sur le plancher buccal antérieur, une tuméfaction régulière, de $4 \mathrm{~cm}$ dans son grand axe, molle et recouverte d'une muqueuse d'aspect normal. Cette tuméfaction refoule la langue vers le côté gauche et engendre des troubles de l'élocution ainsi qu'une gêne à la mastication. Lors de l'examen clinique, on découvre dans la région sous-mentale, une masse bien limitée, arrondie, molle, de $4 \mathrm{~cm}$ de

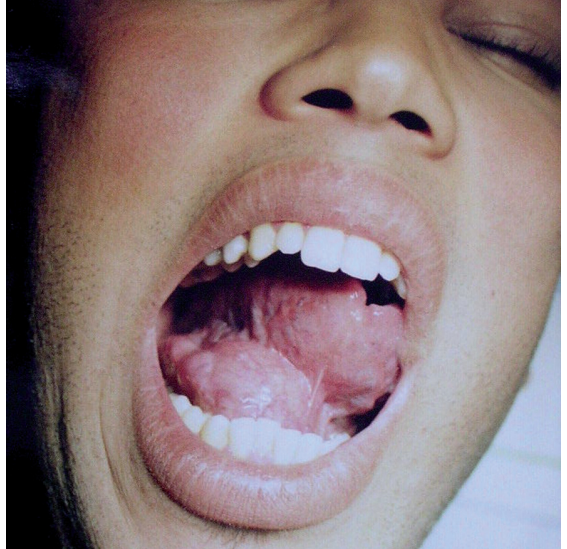

Figure 1: Masse au niveau du plancher buccal refoulant la langue.

Tumour of the oral floor, responsible of a deviation of the tongue.

diamètre, mobile dans les deux plans (Fig. 1). La palpation bidigitale permet de préciser qu'il s'agit d'une masse unique, à expression endobuccale et cervicale. Le reste de l'examen cervico-facial est normal. L'échographie cervicale met en évidence une masse échogène, homogène, bien limitée, occupant la partie paramédiane droite du plancher buccal ; l'examen tomodensitométrique du plancher buccal montre un processus d'allure kystique, comportant des cloisons dans sa partie inférieure, sans atteinte osseuse associée (Fig. 2 et 3). Ces éléments cli-

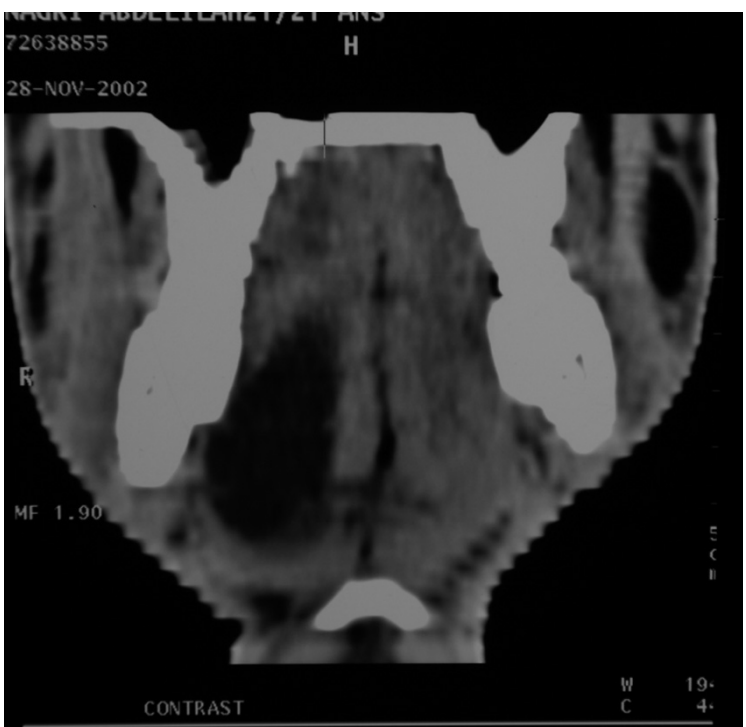

Figures 2 et 3 : Coupes tomodensitométriques axiale et coronale objectivant un processus d'allure kystique, présentant des cloisons à sa partie inférieure, sans atteinte osseuse associée.

Axial and coronal CT scan, showing a cystic tumour, cloisonned, without bone lesion associated. 


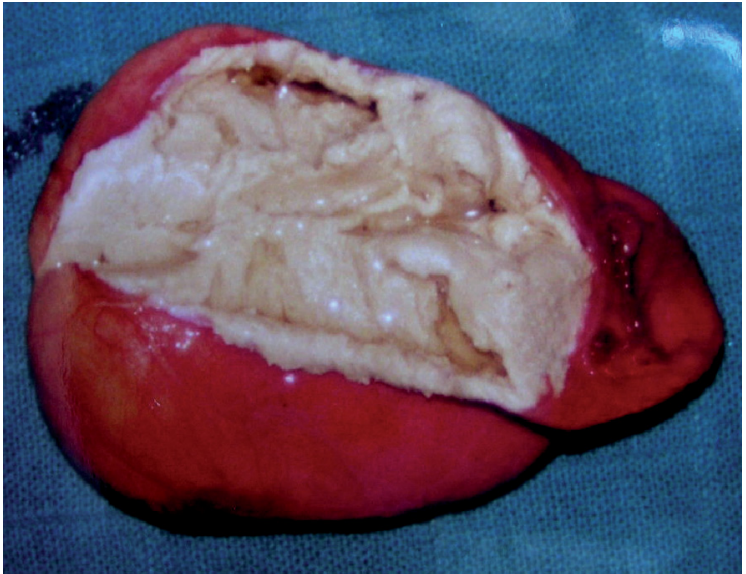

Figure 4 : Kyste oblongue de $5 \mathrm{~cm}$ de grand axe. Après son ouverture on a noté la présence d'un contenu blanchâtre et friable évocateur de débris épidermiques. Oblong cyst of $5 \mathrm{~cm}$ of large axis. After its opening, we found white and friable contents, evocative of epidermis remains.

niques et radiologiques orientent vers le diagnostic de lésion kystique bénigne du plancher buccal, de nature inconnue. Sous anesthésie générale, par voie endobuccale, une exérèse complète en un seul bloc de la lésion kystique du plancher buccal a été réalisée.

A l'examen macroscopique, la pièce opératoire est constituée par un kyste de forme oblongue, de $5 \mathrm{~cm}$ dans son grand axe. Après section, on découvre un contenu blanchâtre, friable, qui semble constitué de débris de kératine (Fig. 4). L'examen microscopique confirme qu'il s'agit d'un kyste épidermoïde : la paroi du kyste est constituée par un épithélium malpighien, pluristratifié, kératinisant, et le contenu est formé par des lamelles de kératine. Le patient est suivi régulièrement à la consultation et aucun signe de récidive tumorale n'a été observé.

\section{COMMENTAIRES}

Le terme de kyste dermoïde est souvent utilisé dans un sens large pour décrire des lésions kystiques pouvant être de nature épidermoïde, dermoïde ou tératoïde ${ }^{[1]}$. Histologiquement, le kyste dermoide vrai comporte un épithélium malpighien, pluristratifié, kératinisant et renfermant des annexes de la peau (glandes sudoripares, glandes sébacées) et des phanères (follicules pileux) [2,3]. En l'absence d'annexes ou de phanères, la lésion est désignée sous le terme de kyste épidermoide ${ }^{[2]}$. Le kyste tératoöde comprend, en plus des éléments spécifiques du kyste dermoïde, des éléments d'origine mésodermique (muscle, os, dents) ${ }^{[1-3]}$.

Le kyste dermoïde est généralement congénital et seulement $10 \%$ semblent être acquis à la suite d'un traumatisme ${ }^{[4]}$. II peut être rencontré, sur la ligne médiane, dans n'importe quelle région de l'organisme ${ }^{[4]}$. La localisation cervico-faciale est rare ; le plus souvent, c'est le plancher buccal qui est le siège de ces kystes. Johnsen et Erich ${ }^{[5]}$, dans une série portant sur 1495 kystes dermoides, ont trouvé que seulement $7 \%$ des cas avaient une localisation cervico-faciale et $23 \%$ de ces derniers siégeaient dans le plancher buccal. Ils peuvent être classés en fonction de leur siège anatomique par rapport aux muscles géniohyoïdiens et mylohyoïdiens (au-dessus, en-dessous, en bissac) ; cette classification a surtout un intérêt pour déterminer la voie d'abord chirurgicale (intra-buccale, extra-buccale ou combinée) [ ${ }^{[6]}$. Cliniquement, le kyste dermoide du plancher buccal, se révèle par une tuméfaction pelvienne ou sous-mentale, non douloureuse, de consistance molle, bien limitée, augmentant progressivement de volume ${ }^{[7,8]}$. L'évolution peut se faire vers une augmentation rapide du volume du kyste, secondaire soit à une sécrétion accrue de sébum ${ }^{[9]}$, soit à une infection du contenu kystique par blocage des glandes sudorifiques, ou par passage des germes de la cavité buccale dans le kyste lors d'un traumatisme [10].

L'échographie est l'examen clinique le plus utile. Elle montre des images homogènes, hypo-échogènes, polykystiques, à bords réguliers ${ }^{[11]}$. Elle est aussi fiable que l'examen tomodensitométrique et l'imagerie par résonance magnétique qui montrent également des images hypodenses multiloculaires [11]. L'apport de l'orthopantomogramme n'est pas contributif sauf si l'on réalise au préalable une injection de produit de contraste dans la cavité kystique [12].

Devant une masse du plancher buccal, indolore, bien limitée, on évoque de nombreuses lésions : pseudokyste salivaire rétentionnel (ranula), kyste du tractus thyréoglosse, neurofibrome, hémangiome, lymphangiome... ${ }^{[12]}$. \begin{tabular}{l}
$\begin{array}{l}\text { médecine } \\
\text { buccale } \\
\text { chirurgie } \\
\text { buccale }\end{array}$ \\
\hline VOL. $12, N^{\circ} 3$ \\
2006
\end{tabular}

page 177 
Les données cliniques et paracliniques permettent d'orienter le diagnostic mais sa confirmation est apportée par l'examen microscopique de la pièce d'exérèse.

Le traitement consiste en l'exérèse chirurgicale complète de la tumeur. Deux voies d'abord sont possibles, endobuccale et cervicale en fonction de la taille et de la localisation de la tumeur ; parfois on doit avoir recours à une voie combinée [13]. Le pronostic est excellent et les récidives sont rares ${ }^{[14]}$. D'exceptionnels cas de transformation maligne survenant sur des kystes dermoïdes ont été rapportés ${ }^{[15]}$. médecine buccale chirurgie buccale

VOL. $12, \mathrm{~N}^{\circ} 3$ 2006

page 178

\section{RÉFÉRENCES}

1 - KING RC, SMITH BR, BURK JL. Dermoid cyst in the floor of the mouth. Review of the literature and case reports. Oral Surg Oral Med Oral Pathol 1985 ; 59 : 578-80.

2 - MAHMOOD S, Moody H. Dermoid, teratorma, choristoma? A rare lesion of the tongue in an adult. $\mathrm{Br} \mathrm{J}$ Oral Maxillofac Surg 2003 ; 41 : 117-9.

3 - ARIYOSHI Y, SHIMAHARA M. Magnetic resonance imaging of a submental dermoid cyst: report of a case. Oral Maxilofac Surg 2003 ; 61: 507-10.

4 - Gold BD, ShEINKOPF DE, LeVy B. Dermoid, epidermoid and teratomatous cysts of the tongue and the floor of the mouth. J Oral Surg 1974 ; 30 : 107-11.

5 - LIPSETT J, SPARNON AL, BYARD RW. Embryogenesis of enterocystomas-enteric duplication cysts of the tongue. Oral Surg Oral Med Oral Pathol 1993 ; 75 : 626-30

6 - MEYER I. Dermoid cysts of the floor of the mouth. Oral Surg Oral Med Oral Pathol $1955 ; 8$ : 1149-64.

7 - SEAH TE, SuFYAN W, SINGH B. Case report of a dermoid cyst at the floor of the mouth. Ann Acad 2004 ; 33 (suppl).
8 - ICHIRO A, SHIGENORI N, TAKEHIKO K, HITOSHI K, IZUMI K. A case of large dermoide cyst in the floor of the mouth. Auris Nasus Larynx 2003 ; 30 : S137-39.

9 - SEWARD GR. Dermoid cysts of the floor of the mouth. Br J Oral Surg $1965 ; 3: 36-47$.

10 - MILAM M, HILL SA, MANALIGOd JM. Lingual delmoid cyst. Otolaryngol Head Neck Surg 2003 ; 128 : 428-9.

11 - Bisschop P, Jortay A, Blondiau P, Van Hoof I, De Roy G. Le kyste dermoïde de la langue. Acta Otorhinolaryngol Belg $1985 ; 39$ : 985-93.

12 - MILES LP, NAIDOO LC, REDDY J. Congenital dermoid cyst of the tongue. J Laryngol Otol 1997 ; 111 : 1179-82.

13 - AL-KhaYAT M, KenYON GS. Midline sublingual dermoid cyst. J Laryngol Otol 1990 ; 104 : 578-80.

14 - MAThuR SK, MENON PRN. Dermoid cyst of the tongue: report of a case. Oral Surg Oral Med Oral Pathol 1980 ; $50: 217-8$.

15 - DEVINE JC, JONES DC. Carcinomatous transformation of a sublingual dermoid cyst: a case report. Int J Oral Maxillofac Surg 2000 ; 29 :126-7. 